\title{
Ultrasonic flaw detection capabilities at first stage of technological process of ship engine overhaul
}

\author{
Alexander Ilyakhinskiy ${ }^{1}$, Vladimir Rodyushkin ${ }^{1, *}$, and Evgeniy Berezin ${ }^{2}$ \\ ${ }^{1}$ Institute of Mechanical Engineering Problems, RAS, 85 Belinskogo street, Nizhniy Novgorod, \\ Russia \\ ${ }^{2}$ Sudoremont Scientific Training Center FSBEI of Higher Education Volga State University of Water \\ Transport, 5 Nesterova street, Nizhniy Novgorod, Russia
}

\begin{abstract}
The technological process of ship engine overhaul begins with dismantling, while the obligatory operation is flaw detection of parts. The flaw detection at this stage involves certain difficulties due to the fact that the entire repair process takes place directly onboard the ship. It is possible to simplify the task of assessing the technical condition of a particular part, if one can assess the performance of this part before dismantling, saving both material assets and time. However, in vessel design, the equipment developers do not pay due attention to ensuring its controllability. The article describes capabilities of ultrasonic flaw detection that can be realized in ship conditions during the first stage of the ship engine overhaul technological process. The ultrasonic technique for estimation of a crankshaft metal damage through scavenging ports of the ship engine is discussed; as well as an option to address the issue of repair or replacement of tap bolts according to their actual technical condition. The described capabilities are due to the uniqueness of ultrasonic flaw detection: the internal space of almost all structural materials can be "observed" by waves of a mechanical nature. Elastic waves, probing the metal, react to all the processes occurring in it, they perform the functions of a universal sensor that delivers information about the controlled environment. They allow to recognize an early stage of defect accumulation - micro damage formation, which is quite natural, since the parameters of elastic waves depend on the density, moduli of elasticity, size and geometry of structural inhomogeneities and other characteristics of the test medium.
\end{abstract}

At present, the operational safety is determined by the criteria based on the presence or absence of material macrodefects such as cracks, shrinkage holes and the like, detectable visually or using standard methods of nondestructive testing. This does not take into account the fundamental fact that during ship operation the microdamage processes occur in the structure of the metal, and the duration of the incubation (latent) periods of these processes is unknown and cannot be determined by classical methods of flaw detection. The service life of ship mechanisms in many respects determines the working capacity of

*Corresponding author: vlkn2005@yandex.ru 
the ship, therefore it is vital to determine the places of possible cracks long before their actual occurrence. Diagnostics of metal fatigue damage is of paramount importance in the procedure of extending the life of worn-out equipment. This is due to the fact that the predicted time to reach the limiting geometric parameters of the crank-shaft necks, determined by instrumental methods, is substantially greater than the predicted time before the defects of fatigue origin occur [1]. The way of extending these time periods was discussed in [2], where the method of metal magnetic memory (MMM), based on the measurement and analysis of the self-magnetic field distribution is involved. However, the absence of a strict physical model, as well as the determination of the applicability limits of the proposed MMM method raise great doubts about unambiguity of the results obtained, besides, the proposed MMM method does not have any metrological support and cannot be offered as a diagnostic method without establishing appropriate metrological certification procedures and applying reliable statistics [3].

Ultrasonic testing in the classic sense, based on the effect of waves reflected from the defect, doesn't need to be conducted for damage control, as there are no physically existing discontinuities yet. Damage is usually understood as reduction in the elastic response of the body due to reduction in the effective area that transfers internal forces from one part of the body to another, which in turn is caused by the appearance and development of a scattered field of microdefects (microcracks in elasticity, dislocations in plasticity, micropores - with creeping, surface microcracks - with fatigue). Not directly measurable damage, i.e. degradation of the mechanical properties of the body, can be detected as a result of analyzing the body's response to various external influences. In particular, in the case of acoustic sounding, external influences consist of ultrasonic pulse generation and reception after its passing through the test medium. Methods of nonlinear acoustics [4] are much more sensitive to the processes of performance degradation of metal than the standard ones. Imperfections in metal structure are largely determined by the nonlinear properties of the medium. By controlling the parameters of the nonlinear medium, which increases when the "nuclei" of the destruction process appear, it is possible to indirectly evaluate the damage.

Since the nonlinearity of the elastic medium leads to the generation of spectral components in the sounding signal at frequencies that are multiple of the fundamental frequency, the level of the spectral component in the probing elastic wave at the doubled frequency of the original signal can be used as a diagnostic sign of metal damage [5]. Taking into account that in the process of maintenance the accumulation of damage initially occurs primarily in the surface layers, and then spreads into the metal mass, the application of surface elastic Rayleigh waves makes it possible to determine more adequately the limiting state of the metal. The possibility of metal damage monitoring with the help of Rayleigh waves was investigated on samples made of St10 metal manufactured in accordance with GOST (National State Standard) 1497, with damage introduced by plastic and low-cycle deformation. The deformations were performed on a universal test machine under the brand of Tinius Olsen Ltd, H100KU model. The maximum limiting load was 100 $000 \mathrm{~N}$. The measurement error of the load was $0.5 \%$. The resolution capability of the system for measuring the displacement of the traverse/sample deformation was $0.001 \mathrm{~mm}$. The acoustic nonlinearity of the sounding signal was monitored using a pair of wedge transducers, which made it possible to generate and detect a tone signal of ultrasonic surface waves. The acoustic nonlinearity for a given damage was monitored by measuring the amplitudes of the first and second partials (1 MHz and $2 \mathrm{MHz}$, respectively). The data obtained are presented in Fig. 1. It can be observed that as the number of loading cycles increases, the acoustic nonlinearity increases as well, and consequently the metal damage. This quantitative information can be useful for resource prediction models and, what is especially important, in the early stages of fatigue. 


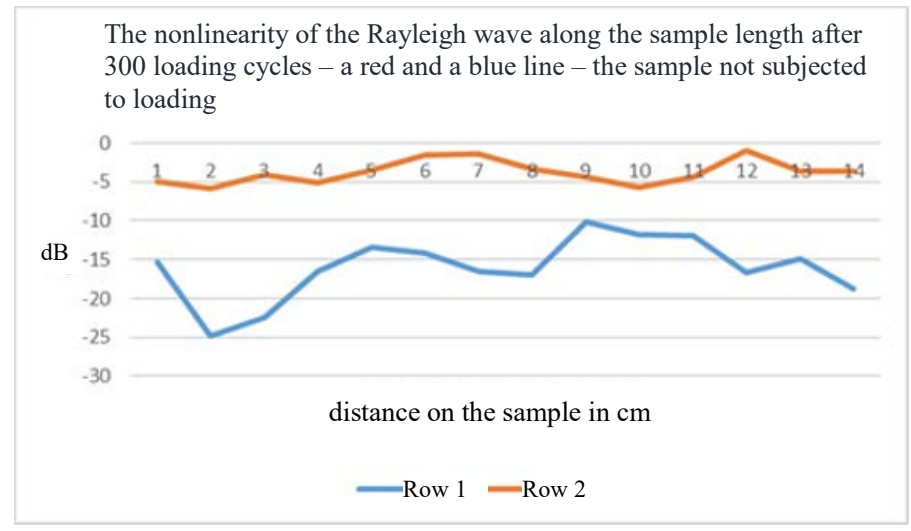

Fig. 1. Results of acoustic nonlinearity measuring for various accumulated damage, obtained for different modes of sample deformation.

To calculate the ratio of the amplitudes of the first and second partials of the sounding signal, the device developed by the authors was used. This device didn't require special radiophysical knowledge [6] and because of its simplicity and compactness allowed from a technical point of view to implement, in ship conditions, a method for estimation of a crank-shaft metal damage through scavenging ports of the ship engine.

In the engine dismantling process, the condition of the tap bolts that fasten together the power unit structure is assessed according to their elongation during the operation cycle. For such an assessment, it is necessary to know the initial length of the tap bolts and only then to decide whether there is an unacceptable elongation that occurred during the depletion of the specified life. If the plastic deformation of the tap bolt is more than $2 \%$, then it must be replaced. It is not always possible to assess the condition of a tap bolt by an instrumental method due to the lack of information about its initial length. The problem can be solved if we use the laws governing surface waves propagation in a deformed metal.

In [7], the possibilities of ultrasonic testing, based on the measurement of the delay (propagation time) of surface waves on a fixed base, or on the results of measuring the nonlinearity of a wave or changing its shape (self-organization coefficient), are investigated. We used the measurement of the delay (propagation time) of surface waves on a fixed base as the simplest and most acceptable diagnostic feature of the degradation processes occurring in the metal, and, consequently, the elongation of the tap bolts that occurred during the operational cycle. The experiments were carried out on flat proportional samples, in accordance with GOST 1497, made of St3 steel $8 \mathrm{~mm}$ thick and a working part $20 \mathrm{~mm}$ wide. The damage was created by plastic deformation of the samples. The surface Rayleigh wave was created by piezoelectric transducers at oscillation frequencies of 2, 5 and $10 \mathrm{MHz}$. The emitting and the receiving transducers were installed at a fixed distance of $50 \mathrm{~mm}$ from each other. The excitation of the emitting transducer was carried out with the help of A1214 flaw detector. From the receiving PET the signal came to the time-interval meter, in place of which an oscilloscope TDS2022 Tektronix with a resolution of $2 \mathrm{nsec}$ was used. The direction of pulse propagation was along the sample. Figure 2 shows the obtained experimental data, showing dependences from the stress-strain deformation volume and the delay (velocity) volume of the elastic Rayleigh wave. 


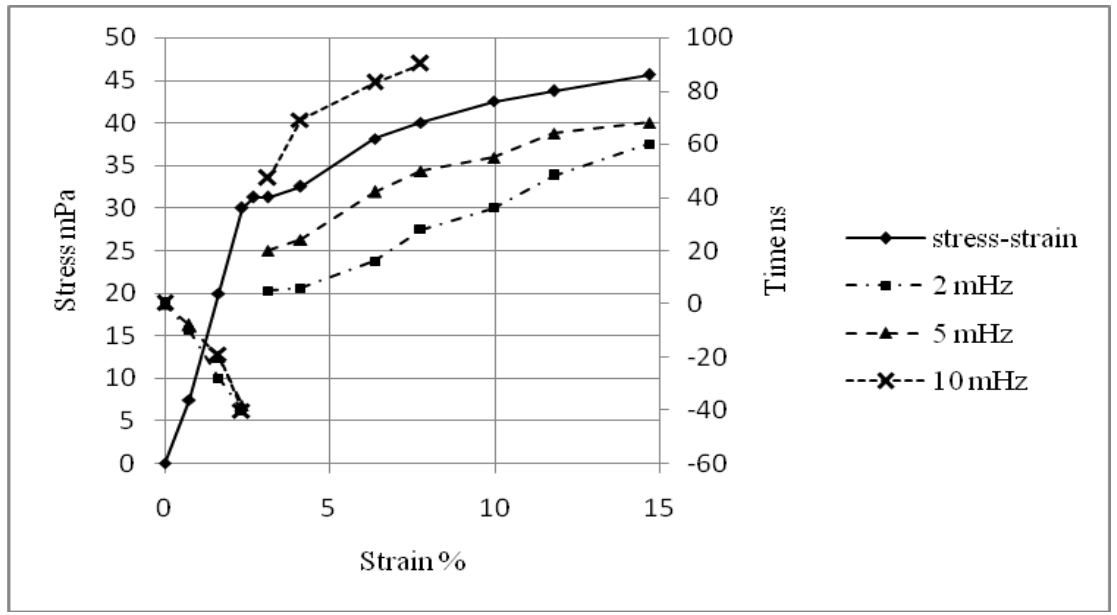

Fig. 2. Dependence of stress-strain and pulse delay of the elastic wave from plastic deformation.

When dealing with plastic deformations (above the yield point: about $2 \%$ deformation, see Fig. 2), there is an obvious fact of the transition of the wave propagation delay from the negative zone to the positive one. The effect of the dependence of the elastic wave velocity on deformations takes a directly opposite sign, in contrast to the elastic region (up to the yield point: about $2 \%$ deformation). It is proposed to use this physical effect as a diagnostic principle in controlling the tap bolt elongation: plastic deformation of more than $2 \%$ in the protruded part of the tap bolt, where the operation of the ship engine (bolting, vibrations, cyclic loads) causes the appearance of residual stresses (deformations) and reflects the reject level. In practice, when dismantling the ship engine and conducting flaw detection, the speed (delay) of the elastic wave pulse is not always known in the absence of loading, which must be taken as zero. In this case, the control technique is based on probing the tap bolt with elastic waves of different frequencies. When frequency dependence of the measured acoustic parameter takes place, the decision is made about the incompatibility of the tap bolt with its safe further operation, since the metal is deformed by a value more than the normative one.

\section{Conclusions}

The article shows the viability of using ultrasonic testing to assess the quality of the ship engine elements in accordance with the accumulated operational damage and the possibility of using it directly onboard the ship at the first stage of the technological repair process.

Using the properties of elastic waves, it is possible to carry out a number of nondestructive testing operations without a complete dismantling of the ship engine components, which makes it possible to optimize the volume of overhaul and reasonably prolong the life of the ship engine parts for the period until the next assessment.

The work was carried out on the topic of the state task of the Institute of Applied Physics of RAS for carrying out fundamental scientific research for the period of 2013-2020. No. 0035-2014-0402 (State Registration No. 01201458047) and partly within the framework of project No. 18-08-00715A of the Russian Foundation for Basic Research. 


\section{References}

1. Khanin S.M., Lysyak I.V., Passumanskiy A.A., Petryanin A.Ya, Manual of Component Wear and Reliability of Diesel Engines Analysis (L.: Transport, 1982)

2. Information portal https://www.korabel.ru/news/comments. - Mironenko I. G., Mironenko A. G., https://www.korabel.ru/news/comments: Reflections on Life Extension of Worn-out Equipment

3. Smorodinskiy Ya. G. Minutes of the Round Table "Method MMM. Purpose, Scope and Assessment of Effectiveness", Territory NDT No.2, P.6-8 (2017)

4. Zaytsev V. Yu., Nazarov V. Ye., Talanov V. I., Nonclassical Manifestations of Microstructural-conditioned Nonlinearity: New Possibilities for Acoustic Diagnostics, UFN, No. 176 :1, P.97-102 (2006)

5. Mishakin V.V., Rodyushkin V.M., Nonlinearity as an Indicator of the Predefective State, Safety of Labor in Industry, No.7. P.48-53 (2009)

6. Vanyagin A.V., Rodyushkin V.M. Measurement of the Damaged Metal Acoustic Nonlinearity, Measuring Technique, No.10. P.42-44 (2017)

7. Yerofeev V.I., Ilyahinskiy A.V., Nikitina Ye.A., Rodyushkin V.M., Ways of Increasing Sensitivity of the Acoustic Sounding Method in Studying Metal Structure, Defectoscopy, No.2. P.11-14 (2018) 